\title{
Generalized Metrizable Spaces, the D-Property And Mappings
}

\author{
a] P.Rajendran b] R.Ragavendran c] Dr.K.Vijayalakshmi \\ [a]Saveetha School of Engineering, Saveetha University, Chennai, India [b] Saveetha \\ School of Engineering, Saveetha University, Chennai, India [c]Saveetha School of Engineering, \\ Saveetha University, Chennai, India
}

\begin{abstract}
The basic properties of D-spaces are discussed and a generalized left separated space is introduced with D-space. Generalized metric spaces that are D-spaces and the behavior of the D-property with respect to the mappings is discussed in this paper.
\end{abstract}

\section{Introduction}

E.K.Van Douwen and W.F.Pfeffer has introduced the concepts of $D$-spaces in 1979. In this paper we show that spaces with additional structure such as a base property or generalized metric property are $D$-spaces.

Definition 1.1.[1]A neighbourhood assignment for a topological space $(X, \tau)$ is a function $N: X \rightarrow \tau$ such that $x \in N(x)$ for each $x \in X$. Xis said to be a $D$-space if for every neighbourhood assignment $N$, there is a closed discrete subset $D$ of $X$ such that $N(x) \backslash x \in D$ covers $X$.

Definition 1.2.[2]We say that the positive cardinality of a family $\gamma$ of sets in $X$ does not exceed a cardinal number $\tau$, if for each $x$ the cardinality of the collection of all members of $\gamma$ containing $x$, does not exceed $\tau$. If the pointwise cardinality of a family does not exceed $\kappa_{0}$, the family is said to be point countable.

Theorem 1.3. Every space $X$ with a point-countable base $B$ is a $D$-space.

Proof: Let $\varphi$ be any arbitrary neighbourhood assignment on . Since $B$ is a base for $X$, for each $x \in X$ we can fix $\psi(x) \in B$ such that $x \in \psi(x) \subset \varphi(x)$. Then $\psi$ is also a neighbourhood assignment on $X$. The family $\psi(x)$ is point countable, since $\psi(x)$ is contained in $B$. By lemma(2.3), there exists a locally finite subset $A$ of $X$ such that $\psi(A)$ covers $\mathrm{X}$. Hence $\varphi(A)$ also covers $\mathrm{X}$. Therefore $X$ is a $D$-space.

Recall that for topological spaces $X, Y f: X \rightarrow Y$ is said to be ans -mapping if fibers of $f$ are seperable.

Corollary1.4. Open continuous $s$-image of a metrizable space is a $D-$ space.

Proof: Since open continuous $s$-image of a metrizable space is a space with a point countable base from theorem(2.4) we have the statement proved.

Corollary1.5.Metrizable spaces are $D$-spaces.

Proof: The Nagata-Smirnov metrization theorem states that "A space $X$ is a metrizable if and only if $X$ is regular and has a countably locally finite basis". Any countably locally finite basis is point countable. Hence by theorem (2.4) $X$ is a $D$-space.I

Definition 1.6.[2] Let $X$ be a topological space. A sequence of open covers $\left\{G_{n}\right\}_{n<\omega}$ is a development for $X$ if $G_{i+1}$ is a refinement for $G_{i}$ and if $x$ is a point in $X, U$ is an open set in $X$ containing $x$, then there is a $k<\omega$ such that $s t\left(x, G_{k}\right) \subset U$ where $s t\left(x, G_{k}\right)=\bigcup\left\{G \in G_{k} / x \in G\right\}$. A topological space $X$ with a development is called a developable space.

Definition 1.7.[4] A Moore space is a regular developable space.

Definition 1.8.[4] A family neighbourhoods $\eta$ of a point $x \in X$ is said to be a neighbourhood base at $x$ if for each neighbourhood $U$ of $x$, there is a $V$ in $\eta$ such that $V \subset U$.

Definition 1.9.[5] Let $X$ be a topological space. A distance function $\rho: X \times X \rightarrow[0, \infty)$ is said to be a semimetric if whenever $x, y \in X$

1. $\rho(x, y) \geq 0$

2. $\quad \rho(x, y)=0$ if and only if $x=y$

3. $\rho(x, y)=\rho(y, x)$.

If $\rho$ is a semimertic on $X$ such that $\left\{S_{\epsilon}(x) / \epsilon>0\right\}$, where $S_{\epsilon}(x)=\{y \in X / \rho(x, y)<\epsilon\}$ is a neighbourhood base at each $x \in X$ then $X$ is called a semimetrizable space.

Theorem 1.10. Every developable space is semimetrizable.

Proof: $X$ be a developable space with a development $\left\{U_{n}\right\}_{n<\omega}$. Then define $\rho$ by $\rho(x, y)=\inf \left\{1 / n / y \in \operatorname{st}\left(x, U_{n}\right)\right\}$. By definition of $\operatorname{st}\left(x, U_{n}\right)$, if $y \in \operatorname{st}\left(x, U_{n}\right)$, then $x \in \operatorname{st}\left(y, U_{n}\right)$. Hence $\rho(x, y)=\rho(y, x)$. Also $\rho(x, y) \geq 0$ whenever $x \neq y . \rho(x, x)=\inf \left\{1 / n / x \in \operatorname{st}\left(x, U_{n}\right)\right\} \rightarrow 0$ as $x \in U_{n}$ for eachn. So $\rho$ is a semimetric on any $x \in X, s_{\epsilon}(x)=\{y \in X / \rho(x, y)<\epsilon\}=\left\{y \in X / \inf \left\{1 / n / y \in \operatorname{st}\left(x, U_{n}\right)\right\}<\epsilon\right\}$. i.e., $s_{\epsilon}(x)$ consists precisely those $y \in \operatorname{st}\left(x, U_{n}\right)$ with $\left\{\inf \left\{1 / n / y \in \operatorname{st}\left(x, U_{n}\right)\right\}<\epsilon\right\}$. Hence for any neighbourhood $U$ of $x$ there exists $\epsilon>0$, 
such that $s_{\epsilon}(x) \subset U$. Hence $\left\{s_{\epsilon}(x) / \epsilon>0\right\}$ is a neighbourhood base at each $x \in X$. Hence $X$ is a semimetrizable space.

Definition 1.11.[5] A topological space $X$ is said to be semistttratifiable if for each open $\operatorname{set} U \subset X$ one can assign a countable sequence $\left\{U_{n}\right\}_{n<\omega}$ of closed subsets of $X$ such that

1. $\mathrm{U}_{n<\omega} U_{n}=U$.

2. $U_{n} \subset V_{n}$ whenever $U \subset V$, where $\left\{V_{n}\right\}_{n<\omega}$ is the sequence assigned to $V$.

Equivalently another definition of a semistratifiable space can be stated as follows: A topological space $X$ is said to be semistratifiable if for each closed set $H \subset X$ One can assign a countable sequence $\left\{U(n, H\}_{n<\omega}\right.$ of open subsets of $X$ such that

1. $\bigcap_{n<\omega} U(n, H)=H$

2. If $K$ is closed with $H \subseteq K$ then $U(n, H) \subseteq U(n, K)$ for all $n<\omega$.

Theorem 1.12. Every semimetrizable space is semistratifiable.

Proof. Let $\mathrm{X}$ be a semimetrizable space with semimetric $\rho$. Let $H$ be a closed subset of $X$ and $U(n, H)=$ $\cup\left\{S_{1 / 2^{n}}(x)^{\circ} / x \in H\right\}$. Then $\{U(n, H)\}_{n<\omega}$ is a countable sequence of open sets in $X$.For any $\in \in H, y \in$ $S_{1 / 2^{n}}(y)^{\circ}$ for each $n<\omega$. Hence $y \in U(n, H)$ for each $n<\omega$. Hence $y \in \bigcap\{U(n, H) / n<\omega\}$. Therefore $H \subseteq$

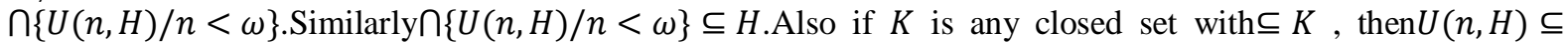
$U(n, K)$ for all $n<\omega$. Hence $X$ is a semistratifiable space.

Theorem 1.13.Semistratifiable spaces are $D$-spaces.

Proof. Let $(X, \tau)$ be a semistratifiable space. To each $W \in \tau$ we can assign a sequence $\{F(W, n)\}_{n<\omega}$ of closed subsets of $X$ such that $W=\cup_{n<\omega} F(W, n)$ and $F(W, n) \subset F(V, n)$ whenever $C$. Let $U: X \rightarrow \tau$ be a neighbourhood assignment in $X$ with range space $\left\{U_{x} / x \in X\right\}$. Let $U_{n}=\left\{U_{x} / x \in F\left(U_{x}, n\right\}\right.$ and $X_{n}=$ $\left\{x \in X / U_{x} \in U_{n}\right\}$. Note that $X=\cup_{j<\omega} X_{j}$. Let $j_{0}$ be the smallest element $<\omega$ such that $U_{j_{0}}$ and $X_{j_{0}} \neq \varnothing$. By transfinite induction pick $U_{x_{\alpha}} \in U_{j_{0}}, \alpha<\gamma_{0}$ such that

1. $\alpha<\beta<\gamma_{0}$ implies $x_{\beta} \notin U_{x_{\alpha}}$.

2. $X_{j_{0}} \subset \mathrm{U}_{\alpha<\gamma_{0}} U_{x_{\alpha}}$.

Let $D_{0}=\left\{x_{\alpha} / \alpha<\gamma_{0}\right\}$. $D_{0}$ is a closed discrete subset of $X$. Pick $z \in \overline{D_{0}}$ note that $z \in \cup_{\alpha<\gamma_{0}} U_{x_{\alpha}}$ because $D_{0} \subset$ $F\left(\mathrm{U}_{\alpha<\gamma_{0}} U_{x_{\alpha}}, j_{0}\right) \subset \mathrm{U}_{\alpha<\gamma_{0}} U_{x_{\alpha}}$. Let $\alpha_{0}$ be the smallest element $<\gamma_{0}$ such that $\in U_{x_{\alpha_{0}}}$. Then $V=U_{x_{\alpha_{0}}} \backslash$ $F\left(\mathrm{U}_{\alpha<\gamma_{0}} U_{x_{\alpha}}, j_{0}\right)$ is a neighbourhood of $z$ such that $V \cap D_{0}=\left\{x_{\alpha_{0}}\right\}$. This shows that $D_{0}$ is closed and discrete. Let $X_{0}^{\prime}=\cup_{\alpha<\gamma_{0}} U_{x_{\alpha}}$ and $j_{1}$ be the smallest element $<\omega$ such that $X_{j_{1}} \backslash X_{0}^{\prime} \neq \emptyset$. Then $\operatorname{let} U_{j_{1}}^{\prime}=\left\{U_{x} \in U_{j_{1}} / x \in\right.$ $\left.X_{j_{1}} \backslash X_{0}^{\prime}\right\}$. Again by transfinite induction, pick $U_{x_{\alpha}} \in U_{j_{1}}^{\prime}, \alpha<\gamma_{1}$ such that

1. $\alpha<\beta<\gamma_{1}$ implies $x_{\beta} \notin U_{x_{\alpha}}$

2. $X_{j_{1}} \backslash X_{0}^{\prime} \subset \mathrm{U}_{\alpha<\gamma_{1}} U_{x_{\alpha}}$

Again we get $D_{1}=\left\{x_{\alpha} / \alpha<\gamma_{1}\right\}$ is a closed discrete subset of $X$ and letting $X_{1}^{\prime}=\bigcup_{\alpha<\gamma_{1}} U_{x_{\alpha}}$ we get that $X_{j_{1}} \subset X_{0}^{\prime} \cup X_{1}^{\prime}$. Hence by ordinary induction we can find integers $j<1, j<2, \ldots$ and closed discrete subsets $D_{i} \subset X_{j_{i}}, i<\omega$ such that

1. Each $X_{j_{i}} \subset \bigcup\left\{U_{x} / x \in \cup_{k=0}^{\prime} D_{k}\right\}$.

2. Each $D_{i+1} \cap\left(\cup\left\{U_{x} / x \in \cup_{k=0}^{\prime} D_{k}\right\}\right)=\emptyset$.

Now letting $D=\bigcup_{i<\omega} D_{i}$. we see that $\left\{U_{x} / x \in D\right\}$ covers $X$ due to (1). To show that $D$ is closed discrete subset of . Pick $z \in X$ and let $n$ be the smallest integer such that $z$ is in some $U_{x_{\alpha}}$ with $x_{\alpha} \in D_{n}$. Then $V=\left(U_{x_{\alpha}} /\right.$ $\left.\bigcup_{k=0}^{n} D_{k}\right) \cup\left\{x_{\alpha}\right\}$ is a neighbourhood of $z$ such that $V \cap D=\left\{x_{\alpha}\right\}$ which shows that $D$ is closed and discrete. Since $D$ is closed and discrete and $\left\{U_{x} / x \in D\right\} \operatorname{covers} X, X$ is a $D$-space.

Definition 1.14.[5] Suppose $X$ is a topological and $d: X \times X \rightarrow[0, \infty)$ such that for all $(x, y) \in X \times X$,

- $d(x, y)=d(y, x)$ and

- $\quad d(x, y)=0$ if and only if $x=y$.

The function $d$ is said to be symmetric for $X$ provided for all non-empty $A \subseteq X, A$ is closed in $X$ if and only if infigid $(x, z) / z \in A\}>0$ for every $x \in X \backslash A$ and $X$ is said to be symmetrizable with symmetric $d$.

Notation 1.15. For $x \in X$ and $n \in N$, let $B(x, 1 / n)=\{z \in X / d(x, z)<1 / n\}$.

Claim 1. $D \subseteq X$.

Claim 2. $W=\{U(x) / x \in D\} \operatorname{covers} X$.

Proof. For any $y \in X$ find $m \in N$ such that $\in I(m)$. If $y \in J(m)$ then $\in U(y) \subseteq U W$. If $y \notin J(m)$ then $y \in(\bigcup\{U(x) / i<m, x \in J(i)\}) \bigcup(\bigcup\{U(x) / x \in J(m), x<y\}) \subseteq \cup W$

Claim 3.Dis a closed discrete set in $U W$ and hence in $X$. 
Proof: This follows if we show that for anyt $\in D, D \backslash\{t\}$ is closed. To this end we may assume $Z=U W$ and let $x \in Z \backslash(D \backslash\{t\})$. It suffices to find a weak neighbourhood $V$ of $x$ such that $V \cap(D \backslash\{t\})=\emptyset$. Let $m$ be the first element of $\mathbb{N}$ such that there is a first elementy of $\mathrm{J}(m)$ where $x \in U(y)$. Now for allz $\in D$ withy $<z$ we have, $z \notin U(y)$. Also for allz $\in D$ with $z<y$ we have, $B(z, 1 / m) \subseteq B\left(x, 1 / k_{2}\right) \subseteq U(z)$ and $x \notin U(z)$, so $z \notin B(x, 1 / m)$. That is, $U(y) \cap B(x, 1 / m)$ is a weak neighbourhood of $x$ with $U(y) \cap B(x, 1 / m) \cap(D \backslash\{y\})=\emptyset$. If $t=y$ claim 3 stands proved.If $\neq y$, then since $y \in D$ we know $x \neq y$ and there is $j \in \mathbb{N}$ such that $y \notin$ $B(x, 1 / j)$. This gives $V=B(x, 1 / j) \cap U(y) \cap B(x, 1 / m)$ as the weak neighbourhood of $x$ with $V \cap(D \backslash\{t\})=\varnothing$ . Hence $D$ is closed and discrete in . Therefore $X$ is a $D$-space.

Corollory 1.16. The quotient compact image of a metrizable space is a $D$-space.

Proof: Since the quotient compact image of a metrizable space $X$ is symmetrizable, $X$ is a $D$-space.

Definition 1.17.[3] A collection of subsets $B=\bigcup\left\{B_{x} / x \in X\right\}$ of a topological space $X$ is said to be a weak base provided

- To each $x \in X$, every member of $B_{x}$ contains $x$

- For any 2 members $B_{1}$ and $B_{2}$ of $B_{x}$ there exists $B_{3} \in B_{x}$ such that $B_{3} \subset B_{1} \cap B_{2}$

- $B$ determines the topology of $X$ in the following way: A set $U \subset X$ is open in $X$ if and only if for allz $\in$ $U$, there exists $B \in B_{z}$ with $B \subseteq U$.

Definition 1.18.[6] A space $X$ is said to be sequential if for every non closed subset $A \subseteq X$ there exists a sequence $\left\{x_{n}\right\}_{n<\omega}$ in $A$ which converges to some $z \in X \backslash A$.

Definition 1.19.[3] If $X$ is a sequential space and $\in W \subseteq X$, we say that $W$ is a weak neighbourhood of $x$ if whenever $\left\{x_{n}\right\}_{n<\omega}$ converges to $x$ then $\left\{x_{n}\right\}_{n<\omega}$ is eventually in $W$.

The next proposition says that in a sequential space the collection of weak neighbourhoods is a weak base for $X$.

Proposition 1.20. If $X$ is a sequential space then a subset $U \subseteq X$ is open if only if for all $x \in U$ there exists a weak neighbourhood $W$ of $x$ such that $W \subseteq U$.

Definition 1.21.[3] A collection $W$ of subsets of a sequential space $X$ is said to be a $\omega$-system for the topology on $X$ if whenever $x \in U \subseteq X$, with $U$ open, there exists a subcollection $V \subseteq W$ such that $x \in \cap V, U V$ is a weak neighbouhood of $x$ and $U V \subseteq U$.

Proposition 1.22. If $f: Z \rightarrow X$ is a quotient map from a space $Z$ onto a $T_{2}$ sequential space $X$ andB is any base for the topology on $Z$ then $W=\{f(B) / B \in \mathbb{B}\}$ is a $\omega$-system for $X$.

Proof.Let $\subseteq X$, with $U$ open. We need to find a subcollection $V \subseteq W$ such that $x \in \cap V, U V$ is a weak neighbourhood of $x$, and $U V \subseteq U$. In $Z$, let $C=\left\{B \in \mathbb{B} / B \cap f^{-1}(x) \neq \varnothing\right.$ and $\left.B \subseteq f^{-1}(U)\right\}$ and let $V=$ $\{f(B) / B \in C\}$. Clearly $x \in \cap V$ and $U V \subseteq U$. To show that $U V$ is a weak neighbourhood of $x$, suppose $\left\{y_{n}\right\}_{n<\omega}$ converges tox; then we need to show that $\left\{y_{n}\right\}_{n<\omega}$ is eventually inUV. If this is not the case there would be a subsequence missing $U V$ completely so without loss of generality we may assume $\left\{y_{n} / n<\omega\right\} \cap(\mathrm{UV})=\emptyset$. Since $X$ is $T_{2}$ a we see that $\left\{y_{n} / n<\omega\right\} \cup\{x\}$ is closed $\operatorname{in} X$ and $f^{-1}\left(\left\{y_{n} / n<\omega\right\} \cup\{x\}\right)$ is closed in $Z$. Now $f^{-1}(x) \subseteq \cup C$, and $\left\{f^{-1}\left(y_{n}\right) / n \in \omega\right\} \cap(\cup C)=\varnothing$ implies that $U\left\{f^{-1}\left(y_{n}\right) / n \in \omega\right\}$ is a closed saturated set in $Z$. This says $\left\{y_{n} / n<\omega\right\}$ is a closed set in $X$, a contradiction. Hence $W=\{f(B) / B \in \mathbb{B}\}$ is a $\omega$-systemfor $X$.

Theorem 1.23. A sequential space $X$ with a point countable $\omega$-system is a $D$-space.

Proof. Let $\mathbb{W}$ be a point countable $\omega$-system for $X$ and for each $x \in X$ let $\mathbb{W}_{x}$ denote $\{W \in \mathbb{W} / x \in W\}$. Suppose $\mathbb{U}=\{U(x) / x \in X\}$ is the range space of neighbourhood assignment $U$ for $X$. For every $x \in X$ pick a subcollection $\mathbb{V}_{x} \subseteq \mathbb{W}_{x}$, such that $x \in \cap \mathbb{V}_{x}$, and $V(x)=U \mathbb{V}_{x}$ is a weak neighbourhood of $x$ and $V(x) \subseteq U(x)$. For $t \in X$, let $\mathbb{H}_{t}$ denote the countable set $\left\{W \in \mathbb{W} / t \in W \in \cup_{x \in X} \mathbb{V}_{x}\right\}$. Consider $\mathbb{H}_{t}$ to be well ordered with an order type as a subset of $\omega$. Identify the centers of elements of $H \in \mathbb{H}_{t}$ by letting

- $c(H)=\left\{x \in H / H \in \mathbb{V}_{x}\right\}$

- $C(t)=\bigcup\left\{c(H) / H \in \mathbb{H}_{t}\right\}$.

By a recursion process we will identify an ordinal $\mu$, countable sets $A_{\alpha} \subseteq X, \alpha<\mu$ and open sets $O_{\alpha}=$ $\mathrm{U}\left\{U(x) / x \in A_{\alpha}\right\}$ so that $\mathrm{U}_{\alpha<\mu} O_{\alpha}=X$ and $D=\mathrm{U}_{\alpha<\mu} A_{\alpha}$ is closed and discrete in $X$. So $\{U(x) / x \in D\}$ would be the subcover of $U$ satisfying $D$-space property.

For an ordinal $\beta$, assuming that $A_{\alpha}$ and $O_{\alpha}$, for all $\alpha<\beta$, have been defined, continue the process as follows: If $\mathrm{U}_{\alpha<\beta} O_{\alpha}=X$, we stop and let $\mu=\beta$.

If $\mathrm{U}_{\alpha<\beta} O_{\alpha} \neq X$, pick some $z_{\beta} \in X \backslash \bigcup_{\alpha<\beta} O_{\alpha}$.

Next we find by induction on $\omega$ an increasing sequence $\left\{F_{n}^{\beta}\right\}_{n \in \omega}$ of finite subsets of $X$, with the initial $F_{0}^{\beta}=$ $\left\{z_{\beta}\right\}$, as follows:

Given that $F_{n}^{\beta}$ is defined and $t \in F_{n}^{\beta}$, let 


$$
R(t)=\left(C(t) \backslash \bigcup_{\alpha \in F_{n}^{\beta}} U(s) \backslash \bigcup_{\alpha<\beta} o_{\alpha}, \quad E_{n}^{\beta}=\left\{t \in F_{n}^{\beta} / R(t) \neq \varnothing\right\}\right.
$$

For $t \in E_{n}^{\beta}$, let $k(t, n)=\min \left\{n,\left|\left\{W \in \mathbb{H}_{t} / R(t) \cap C(W) \neq \emptyset\right\}\right|\right\}$. Now let $W_{t, i}, i=1,2, \ldots, k(t, n)$, be the first $k(t, n)$ elements of $\mathbb{H}_{t}$ such that $R(t) \cap C(W) \neq \varnothing$ and $\operatorname{pick} x(t, i) \in R(t) \cap c\left(W_{t, i}\right)$, for each $i$. We let

$$
F_{n+1}^{\beta}=F_{n}^{\beta} \cup\left\{x(t, i) / t \in E_{n}^{\beta}, 1 \leq i \leq k(t, n)\right\} .
$$

If some $E_{n}^{\beta}=\emptyset$ then $F_{n}^{\beta}=F_{n+1}^{\beta}=F_{n+2}^{\beta}=\cdots$ In any case the resulting $F_{n}^{\beta}, m \in \omega$, form an increasing sequence of finite sets. Now we let $A_{\beta}=\cup_{n \in \omega} F_{n}^{\beta}$.

That concludes the recursion process which defines the countable sets $A_{\alpha} \subseteq X$, for $\alpha<\mu$, and open $\operatorname{sets} O_{\alpha}=$ $\mathrm{U}\left\{U(x) / x \in A_{\alpha}\right\}$. It is clear from the construction that $\mathrm{U}_{\alpha<\mu} O_{\alpha}=X$. The following two observations follow from the construction above:

\section{References}

[1]. E.K. Van Douwen, W.F. Pfeffer, Some properties of the Sorgenfrey line and related spaces, Pacific J.Math. No.2vol.81 (1979), 371377.

[2]. R. Buzyakova, On D-property of strong $\sum$-spaces, comment. Math. Univ. Car. Vol.43 (2002), 493-495.

[3]. D.K. Bruke, Weak bases and D-spaces, Comment.MatUniv.Car.vol.48(2007), 281-289.

[4]. G. Gruenhage, Generalized metric spaces, in the Handbook of set-theoretic topology, K. Kunen and J.E. Vaughan, eds., NorthHolland, Amsterdam, 1984, 423-501.

[5]. G. Gruenhage, A survey of D-spaces, in: L. Balinkostova, A. Caicedo, S.Geschke, M. Scheepers (Eds.), Set Theory and its Applications, Contemp. Math. (2011). 13-28.

[6]. [J.R.Boone, On irreducible spaces, Bull. Austral. Math. Soc. Vol.12(1975), 143-148. 